\title{
Videolaryngoscope for intubation during chest compression
}

\author{
Kun Peng Liu ${ }^{1}$ Fu Shan Xue ${ }^{2}$ Cheng Hui Li ${ }^{1}$
}

Received: 16 June 2015 / Accepted: 17 June 2015 / Published online: 11 July 2015

(C) Japanese Society of Anesthesiologists 2015

\section{To the Editor:}

In a recent manikin study by Okada et al. [1] comparing the performance of the KingVISION (KingV) videolaryngoscope with or without a tube guide blade and Pentax-AWS Airwayscope (AWS) for intubation during chest compressions, the KingV-Guideless videolaryngoscope showed a lower efficacy than the AWS and KingV-Guided device. However, it was unclear whether a stylet was used for intubation with a KingV-Guideless device. The manufacturer recommends that the KingV-Guideless blade requires the use of a stylet shaped to $60-70^{\circ}$ to direct the tracheal tube into the glottis.

A limitation of this study is the lack of a control group in which intubation is performed with the standard Macintosh laryngoscope. A recent manikin study shows that the KingV videolaryngoscope does not prove to be superior to the Macintosh laryngoscope when used for intubation during chest compression [2]. Furthermore, in a randomized controlled clinical trial including 109 patients primarily with prehospital cardiac arrest, the AWS does not show superior efficacy to the Macintosh laryngoscope [3]. As a result, an important question that remains unanswered is whether the KingV device or AWS equals or surpasses the standard tool for intubation during chest compression.

Conflict of interest All authors have no financial support and potential conflicts of interest for this work.

\section{References}

1. Okada D, Komasawa N, Fujiwara S, Minami T. Comparison of tube-guided and guideless videolaryngoscope for tracheal intubation during chest compression in a manikin: a randomized crossover trial. J Anesth 2014; Oct 28. doi:10.1007/ s00540-014-1936-1.

2. Gaszynska E, Gaszynski T. Endotracheal intubation using the Macintosh laryngoscope or KingVision video laryngoscope during uninterrupted chest compression. Biomed Res Int. 2014;2014:250820.

3. Arima T, Nagata O, Miura T, Ikeda K, Mizushima T, Takahashi A, Sakaida K. Comparative analysis of airway scope and Macintosh laryngoscope for intubation primarily for cardiac arrest in prehospital setting. Am J Emerg Med. 2014;32:40-3.

Re: Okada D, et al. Comparison of tube-guided and guideless videolaryngoscope for tracheal intubation during chest compression in a manikin: a randomized crossover trial. J Anesth. 2014 Oct 28.

Fu Shan Xue

xuefushan@aliyun.com; fushan.xue@gmail.com

1 Department of Anesthesiology, China-Japan Friendship Hospital, Beijing, China

2 Department of Anesthesiology, Plastic Surgery Hospital, Chinese Academy of Medical Sciences and Peking Union Medical College, 33 Ba-Da-Chu Road, Shi-Jing-Shan District, Beijing 100144, People's Republic of China 